\title{
A comparison of lobectomy and total thyroidectomy in patients with papillary thyroid microcarcinoma: a retrospective individual risk factor-matched cohort study
}

\author{
Hyemi Kwon', Min Ji Jeon', Won Gu Kim', Suyeon Park', Mijin Kim', \\ Dong Eun Song ${ }^{2}$, Tae-Yon Sung ${ }^{3}$, Jong Ho Yoon ${ }^{3}$, Suck Joon Hong ${ }^{3}$, \\ Tae Yong Kim ', Young Kee Shong' and Won Bae Kim ${ }^{1}$ \\ 'Departments of Internal Medicine, ${ }^{2}$ Pathology, and ${ }^{3}$ Surgery, Asan Medical Center, University of Ulsan \\ College of Medicine, Seoul, Korea
}

Correspondence

should be addressed

to W B Kim

Email

kimwb@amc.seoul.kr

\begin{abstract}
Objective: Papillary thyroid microcarcinoma (PTMC) accounts for most of the increase in thyroid cancer in recent decades. We compared clinical outcomes and surgical complications of lobectomy and total thyroidectomy (TT) in PTMC patients.

Design and methods: In this retrospective individual risk factor-matched cohort study, 2031 patients with PTMC were initially included. Patients who underwent lobectomy or TT were one-to-one matched according to individual risk factors, including age, sex, primary tumor size, extrathyroidal extension, multifocality and cervical lymph node (LN) metastasis.

Results: In total, 688 patients were assigned to each group. During the median 8.5 years of follow-up, 26 patients (3.8\%) in the lobectomy group and 11 patients (1.6\%) in the TT group had recurrences. The relative risk of recurrence was significantly less in the TT than that in the lobectomy group (hazard ratio (HR) $0.41 ; 95 \%$ confidence interval (CI) $0.21-0.81 ; P=0.01)$. Most recurrences $(84.6 \%)$ in the lobectomy group occurred in the contralateral lobe, and all patients were disease-free after completion of thyroidectomy. There were no significant differences in recurrencefree survival between the two groups after exclusion of contralateral lobe recurrences $(\mathrm{HR}, 2.75 ; 95 \% \mathrm{Cl}, 0.08-8.79$; $P=0.08)$. There were significantly more patients with transient and permanent hypoparathyroidism in the TT than that in the lobectomy group $(P<0.001)$.

Conclusions: Lobectomy could be appropriate for most patients with PTMC when there is no evidence of extrathyroidal disease in the preoperative work-up. Preoperative and postoperative imaging studies are important for patients who undergo lobectomy for PTMC, because most recurrences are in the contralateral lobe.
\end{abstract}

\section{Introduction}

The incidence of thyroid cancer has recently increased worldwide. This increase has been predominantly attributed to papillary thyroid microcarcinoma (PTMC) $(1,2,3,4)$. PTMC is defined as a papillary thyroid carcinoma measuring equal or less than $1 \mathrm{~cm}$ in the greatest

www.eje-online.org DOI: 10.1530/EJE-16-0845
두 2017 European Society of Endocrinology Printed in Great Britain dimension (2). PTMC usually has an indolent course and excellent prognosis $(5,6,7,8)$. Disease-specific mortality rate of patients with PTMC who underwent treatment is less than $1 \%$, loco-regional recurrence rate is $2-4 \%$ and distant recurrence rate is $1-2 \%(5,6,7,8)$. The prognostic 
factors for recurrence in patients with PTMC were known such as age, large primary tumor size $(>0.5 \mathrm{~cm})$, presence of extrathyroidal extension, multifocal tumor and the presence of cervical lymph node (LN) metastasis $(5,6,9$, $10,11,12,13,14)$.

Because of its favorable outcomes, previous studies have suggested conservative strategies for evaluating and treating PTMCs $(15,16,17,18,19)$. Recent American Thyroid Association (ATA) guidelines stated that PTMC had a low risk for structural recurrence (20). In one study, among 1235 patients with PTMCs who were underwent active surveillance, $5 \%$ of patients demonstrated increase in size of the tumor and $1.5 \%$ patients showed a novel appearance of LN metastasis on 5-year follow-up (17). ATA guidelines stated if surgery is chosen for patients with small thyroid cancer $(<1 \mathrm{~cm})$ without extrathyroidal extension and clinical No, the initial surgical procedure should be a thyroid lobectomy unless there are clear indications to remove the contralateral lobe (20). A recent retrospective study demonstrated that total thyroidectomy (TT) significantly decreased the risk of overall loco-regional recurrences of PTMC patients $(P<0.001)$ and not the risk of loco-regional recurrence outside of the contralateral remnant lobe $(P=0.6)$ (6). However, there were significant differences in baseline clinicopathological characteristics such as age, sex ratio, primary tumor size and the proportion of extrathyroidal extension, multifocal tumors and LN metastasis between lobectomy and TT patients (6). To the best of our knowledge, no study has compared lobectomy and TT in PTMC patients with similar baseline clinical and pathological risk factors.

In this study, we aimed to compare the clinical outcomes and surgical complications in patients with PTMC according to surgical extent (lobectomy and TT) after individual risk factor matching. We applied a oneto-one matching method to control the risk factors that may affect the prognosis of PTMC and to minimize the selection bias in decisions for surgical extent.

\section{Subjects and methods}

\section{Study design and patients}

This retrospective cohort study consecutively included 2031 patients with PTMC who underwent initial thyroid surgery and routine prophylactic central compartment node dissection from 1998 to 2007 at Asan Medical Center in Seoul, Korea. Ninetynine patients with lateral cervical LN metastasis (N1b) and 4 patients with distant metastasis (M1) at initial diagnosis were excluded. Of the 2031 patients, 755 (32\%) underwent lobectomy, whereas 1276 (63\%) underwent TT (Table 1). Patients who underwent lobectomy or TT were one-to-one matched according to individual risk factors including age, sex, primary tumor size, extrathyroidal extension, multifocality and central compartment LN metastasis (pN1a). Patients within 3 years of age were considered to be the same age. Primary tumors with size differences of less than $0.2 \mathrm{~cm}$ were considered to be the same size for oneto-one matching. As a result of individual risk factor matching, 688 patients were assigned to each group. We compared the clinical outcomes of 1376 patients with PTMC according to surgical extent. Clinicopathological features of PTMC patients who were excluded from the individual matching were described in Supplementary Table 2 (see section on supplementary data given at the end of this article). This study was approved by the Institutional Review Board of Asan Medical Center.

Table 1 Baseline clinicopathological features of patients with PTMC according to surgical extent. Continuous variables are presented as medians (inter-quartile range). Categorical variables are presented as numbers with percentages.

\begin{tabular}{|c|c|c|c|c|c|}
\hline & \multirow[b]{2}{*}{$\begin{array}{l}\text { Lobectomy } \\
\quad(n=755)\end{array}$} & \multirow[b]{2}{*}{$\begin{array}{l}\text { Total thyroidectomy } \\
\qquad(n=1276)\end{array}$} & \multirow[b]{2}{*}{$P$ value } & \multicolumn{2}{|c|}{ After individual matching } \\
\hline & & & & $\begin{array}{l}\text { Lobectomy } \\
\qquad(n=688)\end{array}$ & $\begin{array}{c}\text { Total thyroidectomy } \\
\qquad(n=688)\end{array}$ \\
\hline Age (year) & $47(40-53)$ & $50(42-56)$ & $<0.001^{a}$ & $47(41-54)$ & $47(41-54)$ \\
\hline$\geq 45$ year & $423(55)$ & $855(67)$ & $<0.001^{\mathrm{b}}$ & $405(59)$ & $415(60)$ \\
\hline $\operatorname{Sex}(F)$ & $672(89)$ & $1118(88)$ & $0.387^{b}$ & $628(91)$ & $628(91)$ \\
\hline Primary tumor size (cm) & $0.6(0.5-0.8)$ & $0.7(0.5-0.8)$ & $<0.001^{a}$ & $0.6(0.5-0.8)$ & $0.6(0.5-0.8)$ \\
\hline$>0.5 \mathrm{~cm}$ & $451(60)$ & $885(69)$ & $<0.001^{\mathrm{b}}$ & $415(60)$ & $435(63)$ \\
\hline Extrathyroidal extension (Y) & $274(36)$ & $621(49)$ & $<0.001^{\mathrm{b}}$ & $261(38)$ & $261(38)$ \\
\hline Multifocality (Y) & $79(10)$ & $309(24)$ & $<0.001^{\mathrm{b}}$ & $69(10)$ & $69(10)$ \\
\hline Cervical LN metastasis (Y) & $212(28)$ & $403(32)$ & $0.107^{b}$ & $178(26)$ & $178(26)$ \\
\hline
\end{tabular}

www.eje-online.org 


\section{Management and follow-up protocol}

The protocol was shared at our center to maintain consistent treatment and follow-up for all patients, as previously reported $(5,21,22)$. Routine preoperative neck ultrasonography (US) was done in all the patients. If they had indeterminate or suspicious thyroid nodules in contralateral lobe, the nodules were evaluated by US-guided fine-needle aspiration cytology (FNAC) before thyroid surgery. The surgical extent was determined depending on patient preference and the decision of the surgeon at the time of surgery. Routine prophylactic ipsilateral central compartment LN dissection was performed for patients who underwent lobectomy. Prophylactic ipsilateral or bilateral central compartment LN dissection was performed for patients who underwent total thyroidectomy. Therapeutic neck dissection was performed when metastatic cervical LNs were confirmed preoperatively or suspicious metastatic LNs were detected at the time of surgery. Subsequent radioactive iodine (RAI) remnant ablation therapy was conducted for some patients with PTMC who underwent TT. After initial therapy, patients who were underwent total thyroidectomy took levothyroxine for TSH suppression. In lobectomy group, 262 patients (35\%) took levothyroxine for TSH suppression, and 70 patients (9\%) took levothyroxine for substitutive therapy. All patients were regularly followed up with physical examinations, thyroid function tests, serum thyroglobulin (Tg) and anti-Tg antibody measurement every 6-12 months for at least 2 years after surgery. Neck US was performed during the first 6-12 months after initial therapy and repeated thereafter at 12- to 24-month intervals. Diagnostic wholebody scans (WBS) were performed after TT and RAI remnant ablation. Additional diagnostic imaging studies, such as computed tomography (CT), magnetic resonance imaging (MRI) or whole-body fluoro-deoxyglucose (FDG)positron emission tomography (PET) were performed to detect recurrence or distant metastasis in some patients.

\section{Definitions}

Loco-regional recurrence was defined as pathologically or cytologically proven lesions based on imaging studies. Recurrence-free survival (RFS) was defined as the time interval between initial surgery and the detection of structural persistent/recurrent disease. Transient hypoparathyroidism was defined as postoperative hypocalcemia with albumin-corrected serum total calcium $<8.0 \mathrm{mg} / \mathrm{dL}(2.0 \mathrm{mmol} / \mathrm{L})$ and/or the need for calcium/vitamin D supplements to maintain serum calcium within the reference range or to prevent symptoms $(23,24)$. Permanent hypoparathyroidism was defined as persistent hypocalcemia 12 months after surgery requiring calcium and vitamin D supplements (23).

\section{Statistical analysis}

For individual risk factor matching and analysis, Greedy method was used by SAS version 9.4 (SAS Institute Inc., Cary, NC, USA). Several statistical analyses were conducted using SPSS, version 21.0 (SPSS). Graphs were produced using Prism, version 5.01 (GraphPad Software). Continuous variables are presented as medians with inter-quartile ranges (IQR). Categorical variables are presented as numbers with percentages. The Mann-Whitney $U$ test was used to compare continuous variables. The chi-square test and Fisher's exact test were used to compare categorical variables. In the matched data set, risks of recurrence were compared using Cox regression models, with robust standard errors that accounted for the clustering of matched pairs. The Cox proportional hazards model with hazard ratios (HRs) and 95\% confidence intervals (CIs) was used to evaluate risks of recurrence according to surgical extent. RFS curves were constructed using the Kaplan-Meier method, and the log-rank test was used to evaluate differences in the RFSs between groups. $P$ values $<0.05$ were considered statistically significant. All $P$ values were two-sided.

\section{Results}

\section{Baseline characteristics before individual matching}

The baseline characteristics of the 2031 PTMC patients according to surgical extent are listed in Table 1. The median age of the lobectomy group was significantly younger than that of the TT group $(P<0.001)$. There were no statistically significant differences in sex ratio between the two groups. Median primary tumor size in the lobectomy group was significantly smaller than that in the TT group $(0.6 \mathrm{~cm}$ vs $0.7 \mathrm{~cm}, P<0.001)$. The proportions of extrathyroidal extension and multifocality were significantly higher in the TT group than that in the lobectomy group. Extrathyroidal extension was present in 274 patients in the lobectomy group (36\%) and 621 patients $(49 \%)$ in the TT group $(P<0.001)$. Patients with multifocal tumors comprised $10 \%$ and $24 \%$ in the lobectomy and TT groups respectively $(P<0.001)$. 
There were no significant differences in the proportion of patients with cervical LN metastasis.

\section{Clinical outcomes according to each risk factor before individual matching}

The clinical outcomes of PTMC patients were evaluated according to each risk factor (Table 2). In univariate analyses, there were no significant associations between recurrence rates and surgical extent, primary tumor size and extrathyroidal extension. However, older patients had fewer recurrences than younger patients (HR: 0.96, 95\% CI: $0.93-0.98 ; P<0.001)$. Female had fewer recurrences than male (HR: 0.39, 95\% CI: 0.23-0.68; $P=0.001$ ). Multifocal tumor (HR: $1.85,95 \%$ CI: $1.11-3.08 ; P=0.02$ ) and cervical LN metastasis (HR: 2.32, 95\% CI: 1.45-3.71; $P<0.001$ ) were associated with more recurrences of PTMC. In multivariate analysis models 1 and 2, TT was not associated with the risk of recurrence. Younger age, male gender, multifocality and cervical LN metastasis were independent risk factors for recurrences in patients with PTMC. These variables were included in individual risk factor matching.

\section{Clinicopathological features and clinical outcomes according to surgical extent after individual risk factor matching}

Patients who underwent lobectomy or TT were one-toone matched according to individual risk factors. The baseline characteristics of 688 patients in each group were similarly adjusted (Table 1 ). The median age was 47 years, and the median primary tumor size was $0.6 \mathrm{~cm}$. In all, $38 \%$ of patients had extrathyroidal extension, $10 \%$ patients had multifocal PTMCs and 26\% had cervical LN metastasis. After individual matching, the clinical outcomes of the patients were evaluated according to surgical extent. During the median 8.5 years of follow-up, 26 patients (3.8\%) in the lobectomy group and 11 patients (1.6\%) in the TT group had recurrences (Table 3). There were significantly fewer recurrences in the TT group than that in the lobectomy group (HR: $0.41,95 \%$ CI: $0.21-$ $0.81 ; P=0.01)$.

Most recurrences $(84.6 \%, 22$ of 26 patients) in the lobectomy group occurred in the contralateral lobe (Table 3 and Supplementary Table 1). The median duration between the initial thyroid surgery and the detection of recurrence was 4.4 years. In the lobectomy group, only 4 recurrences occurred in lateral cervical LNs. In the TT group, 1 patient had a recurred disease at operation bed and the remaining 10 patients had
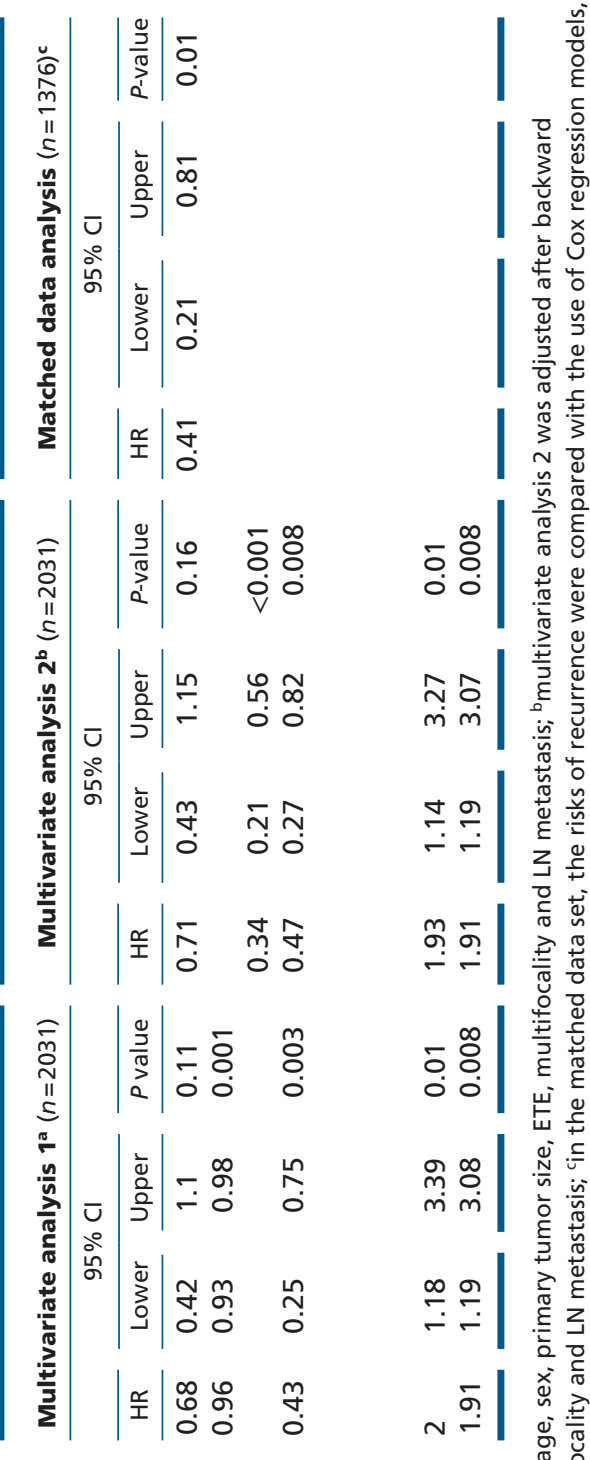

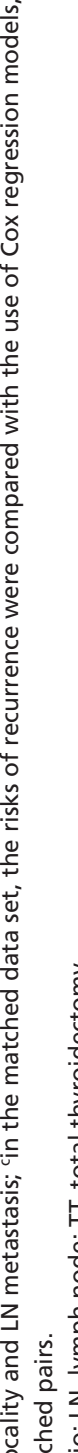

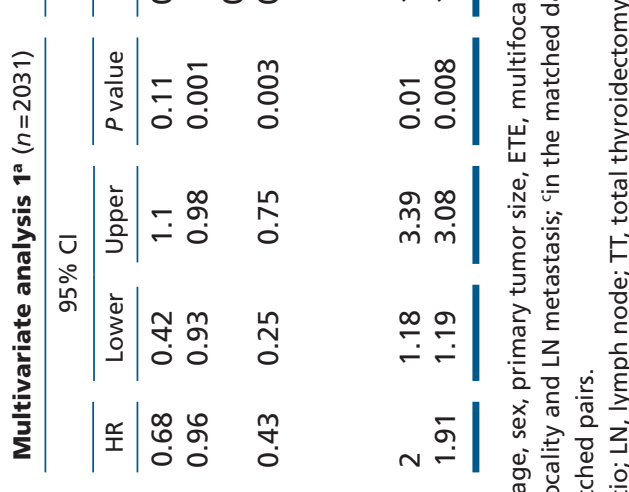

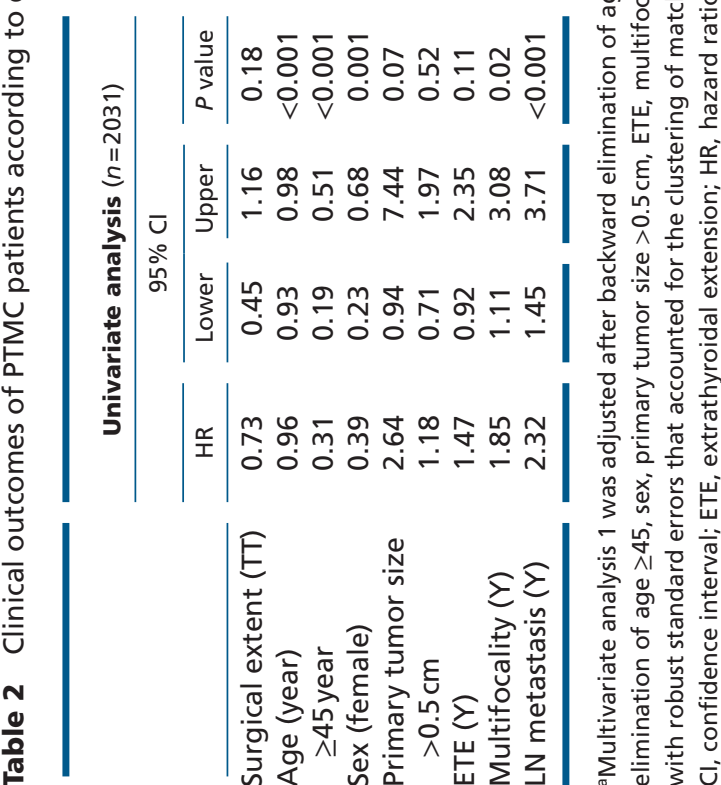

www.eje-online.org 
Table 3 Types of recurrences in PTMC patients $(n=1376)$ according to surgical extent.

\begin{tabular}{|c|c|c|}
\hline Type of recurrence & $\begin{array}{l}\text { Lobectomy } \\
\qquad(n=688)\end{array}$ & $\begin{array}{c}\text { Total } \\
\text { thyroidectomy } \\
(n=688)\end{array}$ \\
\hline Total recurrences $(n)$ & 26 & 11 \\
\hline Contralateral lobe $(n)$ & 22 & 0 \\
\hline Median durationa (year) (IQR) & $4.4(3.0-5.9)$ & \\
\hline Operation bed $(n)$ & 0 & 1 \\
\hline Median durationa (year) (IQR) & & 5.0 \\
\hline Lateral cervical LN $(n)$ & 4 & 10 \\
\hline Median durationa (year) (IQR) & $2.9(1.5-4.4)$ & $2.7(1.6-3.2)$ \\
\hline
\end{tabular}

a Median duration was defined as the median time interval between the initial surgery and the detection of structural persistent/recurrent disease. IQR, inter-quartile range; LN, lymph node.

lateral cervical LN recurrences (Supplementary Table 1). All patients with recurrences remained in a disease-free state after completion thyroidectomy and/or therapeutic neck dissection during a median 5.7 years (IQR 3.7-7.8) of follow-up.

In the lobectomy group, 122 patients (18\%) had bilateral nodules in preoperative neck US. The proportion of bilateral nodules in patients with recurrence was $23 \%$ (6 of 26 ) and $18 \%$ (116 of 662 ) in patients without recurrence. There was no significant difference in bilateral nodules between patients who underwent lobectomy with or without recurrence $(P=0.5)$.

Sixty-nine of the 688 patients (10\%) who were treated with lobectomy had multifocal PTC in pathological analysis of surgical specimen. Twenty-six of 688 patients had recurrent PTC and only two patients with multifocal PTC had recurrent PTC in our study. There was no significant correlation between multifocality and recurrence in lobectomy group $(P=0.7)$.

\section{Recurrence-free survival according to surgical extent}

Patients who underwent TT had better RFS than those who underwent lobectomy ( $P=0.01$; Fig. $1 \mathrm{~A})$. We also
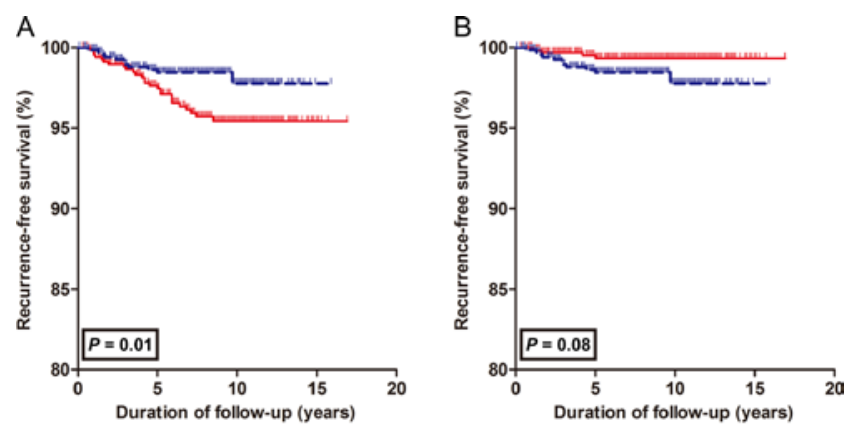

$$
\begin{aligned}
& -- \text { Total thyroidectomy group } \\
& + \text { Lobectomy group }
\end{aligned}
$$

\section{Figure 1}

(A) Recurrence-free survival (RFS) according to surgical extent. Patients with PTMC who underwent total thyroidectomy demonstrated improved RFS compared to patients who underwent lobectomy $(P=0.01)$. (B) RFS according to surgical extent after the exclusion of patients with contralateral lobe recurrence who were disease free after completion thyroidectomy. There were no significant differences in RFS according to surgical extent after the exclusion of contralateral lobe recurrences. $P$ values were determined using the log-rank test. A full colour version of this figure is available at $h$ ttp://dx.doi.org/10.1530/EJE-16-0845.

compared RFSs according to surgical extent, after the exclusion of contralateral lobe recurrences, which were the most types of recurrence in the lobectomy group. There were no significant differences in RFSs between the two groups (HR: 2.75, 95\% CI: 0.08-8.79; $P=0.08$; Fig. 1B).

\section{Complications according to surgical extent}

We compared the complication rates of the lobectomy and TT groups (Table 4). There were no significant differences in postoperative bleeding/hematoma or recurrent laryngeal nerve injury in both groups before and after individual risk factor matching.

\begin{tabular}{|c|c|c|c|c|c|c|}
\hline & \multirow[b]{2}{*}{$\begin{array}{l}\text { Lobectomy } \\
\qquad(n=755)\end{array}$} & \multirow[b]{2}{*}{$\begin{array}{l}\text { Total thyroidectomy } \\
\qquad(n=1276)\end{array}$} & \multirow[b]{2}{*}{ P-value $^{a}$} & \multicolumn{3}{|c|}{ After individual matching } \\
\hline & & & & $\begin{array}{l}\text { Lobectomy } \\
\qquad(n=688)\end{array}$ & $\begin{array}{l}\text { Total thyroidectomy } \\
\qquad(n=688)\end{array}$ & $P$-value ${ }^{a}$ \\
\hline Total complications & $8(1)$ & $213(17)$ & $<0.001$ & $7(1)$ & $124(18)$ & $<0.001$ \\
\hline Bleeding & $7(1)$ & $13(1)$ & 0.8 & $5(0.7)$ & $7(1)$ & 0.6 \\
\hline Hypoparathyroidism & 0 & $193(15)$ & $<0.001$ & 0 & $115(17)$ & $<0.001$ \\
\hline Transient & 0 & $170(13)$ & & 0 & $103(15)$ & \\
\hline Permanent & 0 & $23(1.8)$ & & 0 & $12(1.7)$ & \\
\hline RLN injury & $2(0.3)$ & $12(0.9)$ & 0.1 & $2(0.3)$ & $4(0.6)$ & 0.7 \\
\hline
\end{tabular}

Table 4 Surgical complications according to surgical extent. 
However, patients who underwent TT had significantly more transient (103 of 688 patients, 15\% after matching) and permanent (12 of 688 patients, $1.7 \%$ after matching) hypoparathyroidism $(P<0.001)$.

\section{Discussion}

In this retrospective matched cohort study, we evaluated the clinical outcomes and surgical complications of PTMC patients according to surgical extent. Patients who underwent lobectomy or TT were one-to-one matched according to individual risk factors, including age, sex, primary tumor size, extrathyroidal extension, multifocality and central compartment LN metastasis (pN1a). TT for patients with PTMC improved RFS $(P=0.01)$. However, patients who underwent TT had more transient and permanent hypoparathyroidism. Most recurrences $(84.6 \%, 22$ of 26 patients) in patients who underwent lobectomy occurred in the contralateral lobe of the thyroid, and these patients remained disease-free after completion thyroidectomy. After the exclusion of contralateral lobe recurrences, there were no significant differences in RFSs between the lobectomy and TT groups. These findings suggest that lobectomy is an appropriate surgical extent for most patients with PTMC when clinical cervical LN metastasis is excluded in preoperative imaging studies. Preoperative and postoperative imaging studies are very important when thyroid lobectomy is applied to patients with PTMC.

Most patients with PTMC demonstrated excellent clinical outcomes, and all PTMC patients with recurrences could reach a disease-free state after re-operation. These findings are consistent with previous studies $(5,6,7,8$, $10,11,12)$. In multivariate analyses, younger age, male gender, multifocality and cervical LN metastasis were related to recurrence of PTMC, as previously reported $(5,6,9,10,11,12,13,14)$. The median age of the TT group was significantly older, and the median primary tumor size was significantly larger than those of the lobectomy group $(P<0.001)$. Extrathyroidal extension, multifocality and central cervical LN metastasis (N1a) were also more frequent in the TT group. These differences in baseline clinicopathological features between the two groups were the main limitation to the appropriate comparison of surgical extent. In general, more extensive surgical approach tends to be selected for patients with more risk factors. However, this study was able to overcome this limitation by using the individual risk factor matching method to compare clinical outcomes according to surgical extent in balanced subjects.

In this study, TT for patients with PTMC was able to improve RFS $(P=0.01)$. However, there were no significant differences in RFS between the lobectomy and TT group after the exclusion of contralateral lobe recurrences. Several retrospective studies have suggested that TT can improve survival rates, decrease recurrence rates, make use of RAI remnant ablation and facilitate follow-up with diagnostic WBS and serum $\operatorname{Tg}(25,26,27)$. However, recent studies have demonstrated that clinical outcomes of lobectomy are not inferior to those of TT $(20,28,29$, 30). A study using the SEER database demonstrated no difference in 10-year overall survival (90.8\% for lobectomy vs $90.4 \%$ for TT) or 10-year cancer-specific survival ( $98.6 \%$ for lobectomy vs $96.8 \%$ for TT) between lobectomy and TT (29). For these reasons, the surgical management paradigm has moved away from TT toward conservative strategies such as thyroid lobectomy for patients with low-risk PTMC (20).

Most recurrences in the lobectomy group occurred in the contralateral lobe. All the study patients underwent preoperative US, and indeterminate or suspicious thyroid nodules in contralateral lobe were evaluated by US-guided FNAC before surgery. Median duration for detection of recurrence was 4.4 years. Considering that we routinely followed up the patients every 6-12 months with neck US, the recurrent lesions should be newly developed after initial surgery.

The surgical risks of TT are known to be significantly higher than those of lobectomy from previous studies $(31,32)$. In a recent meta-analysis, the relative risk (RR) of hemorrhage/hematoma after TT was 2.6 (31). There were more transient $(\mathrm{RR}=10.7)$ and permanent $(\mathrm{RR}=3.2)$ hypocalcemia and recurrent laryngeal nerve injuries $(\mathrm{RR}=1.9)$ in patients who underwent TT (31). Even highvolume surgeons have more postoperative complications when performing TT than performing lobectomy (32). Considering that the fundamental goals of treatment for patients with differentiated thyroid cancer are to improve survival and reduce recurrence/persistence as well as to minimize treatment-related morbidity, it is also important to reduce the complications associated with surgery (20).

This study had several limitations. There was the possibility of selection bias due to its retrospective nature and the involvement of only our single tertiary referral center. For individual risk factor matching, we excluded 67 patients in the lobectomy group and 588 patients in TT group (Supplementary Table 2). This approach may 
lead to a substantial error. Because we included patients from the early 2000 s, about $63 \%$ of patients underwent TT. Among them, $87 \%$ of patients received RAI remnant ablation with median dose of $2.8 \mathrm{GBq}$ (1.1-5.6). We were not able to evaluate the effects of RAI remnant ablation on clinical outcomes. However, all recurrences in our matched cohort were detected by regular neck US. The influences of serum Tg measurement after TT and remnant ablation were minimized for the detection of recurrent disease in our study. In addition, prophylactic central compartment LN dissection was performed in our study patient, and we could have definitely known the pathological $\mathrm{N}$ staging and used this information for risk factor matching in the two groups. Notably, this study is the first to compare clinical outcomes and surgical complications in PTMC patients managed by a uniform protocol at a single institution according to surgical extent applying an individual risk factor matching method.

In conclusion, most patients with PTMC demonstrated excellent clinical outcomes. Lobectomy could be an appropriate surgical extent for most patients with PTMC when there is no evidence of bilateral tumors or extrathyroidal disease in preoperative work-up. Most recurrences in patients who underwent lobectomy occurred in the contralateral lobe. There was no difference in RFS between the lobectomy and TT groups after the exclusion of patients with contralateral lobe recurrences who were disease free after completion thyroidectomy. Preoperative and postoperative imaging studies are important for patients who undergo lobectomy for PTMC because most recurrences are in the contralateral lobe.

Supplementary data

This is linked to the online version of the paper at http://dx.doi.org/ 10.1530/EJE-16-0845.

Declaration of interest

The authors declare that there is no conflict of interest that could be perceived as prejudicing the impartiality of the research reported.

Funding

This study was supported by a grant from the Korean Health Technology R\&D project, Ministry of Health \& Welfare, Republic of Korea (HC15C3372). This study was presented at a Young Investigator Session of the 39th annual meeting of the European Thyroid Association in Copenhagen, Denmark.

\section{References}

1 Cho BY, Choi HS, Park YJ, Lim JA, Ahn HY, Lee EK, Kim KW, Yi KH, Chung JK, Youn YK et al. Changes in the clinicopathological characteristics and outcomes of thyroid cancer in Korea over the past four decades. Thyroid 201323 797-804. (doi:10.1089/thy.2012.0329)

2 Davies L \& Welch HG. Increasing incidence of thyroid cancer in the United States, 1973-2002. JAMA 2006295 2164-2167. (doi:10.1001/ jama.295.18.2164)

3 Elisei R, Molinaro E, Agate L, Bottici V, Masserini L, Ceccarelli C, Lippi F, Grasso L, Basolo F, Bevilacqua $\mathrm{G}$ et al. Are the clinical and pathological features of differentiated thyroid carcinoma really changed over the last 35 years? Study on 4187 patients from a single Italian institution to answer this question. Journal of Clinical Endocrinology and Metabolism 201095 1516-1527. (doi:10.1210/jc.2009-1536)

4 McNally RJ, Blakey K, James PW, Gomez Pozo B, Basta NO \& Hale J. Increasing incidence of thyroid cancer in great Britain, 1976-2005:age-period-cohort analysis. European Journal of Epidemiology 201227 615-622. (doi:10.1007/s10654-012-9710-x)

5 Jeon MJ, Kim WG, Choi YM, Kwon H, Lee YM, Sung TY, Yoon JH, Chung KW, Hong SJ, Kim TY et al. Features predictive of distant metastasis in papillary thyroid microcarcinomas. Thyroid 201626 161-168. (doi:10.1089/thy.2015.0375)

6 Kim SK, Park I, Woo JW, Lee JH, Choe JH, Kim JH \& Kim JS. Total thyroidectomy versus lobectomy in conventional papillary thyroid microcarcinoma: analysis of 8676 patients at a single institution. Surgery 2017161 485-492. (doi:10.1016/j.surg.2016.07.037)

7 Siddiqui S, White MG, Antic T, Grogan RH, Angelos P, Kaplan EL \& Cipriani NA. Clinical and pathologic predictors of lymph node metastasis and recurrence in papillary thyroid microcarcinoma. Thyroid 201626 807-815. (doi:10.1089/thy.2015.0429)

8 Yu XM, Wan Y, Sippel RS \& Chen H. Should all papillary thyroid microcarcinomas be aggressively treated? An analysis of 18 445 cases. Annals of Surgery 2011254 653-660. (doi:10.1097/ SLA.0b013e318230036d)

9 Mercante G, Frasoldati A, Pedroni C, Formisano D, Renna L, Piana S, Gardini G, Valcavi R \& Barbieri V. Prognostic factors affecting neck lymph node recurrence and distant metastasis in papillary microcarcinoma of the thyroid: results of a study in 445 patients. Thyroid 200919 707-716. (doi:10.1089/thy.2008.0270)

10 Pazaitou-Panayiotou K, Capezzone M \& Pacini F. Clinical features and therapeutic implication of papillary thyroid microcarcinoma. Thyroid 200717 1085-1092. (doi:10.1089/thy.2007.0005)

11 Pisanu A, Saba A, Podda M, Reccia I \& Uccheddu A. Nodal metastasis and recurrence in papillary thyroid microcarcinoma. Endocrine 2015 48 575-581. (doi:10.1007/s12020-014-0350-7)

12 Pyo JS, Sohn JH \& Kang G. Detection of tumor multifocality is important for prediction of tumor recurrence in papillary thyroid microcarcinoma: a retrospective study and meta-analysis. Journal of Pathology and Translational Medicine 201650 278-286. (doi:10.4132/jptm.2016.03.29)

13 Ross DS, Litofsky D, Ain KB, Bigos T, Brierley JD, Cooper DS, Haugen BR, Jonklaas J, Ladenson PW, Magner J et al. Recurrence after treatment of micropapillary thyroid cancer. Thyroid 200919 1043-1048. (doi:10.1089/thy.2008.0407)

14 Chow SM, Law SC, Chan JK, Au SK, Yau S \& Lau WH. Papillary microcarcinoma of the thyroid-prognostic significance of lymph node metastasis and multifocality. Cancer 200398 31-40. (doi:10.1002/ cncr.11442)

15 Ahn HS, Kim HJ \& Welch HG. Korea's thyroid-cancer "epidemic": screening and overdiagnosis. New England Journal of Medicine 2014 371 1765-1767. (doi:10.1056/NEJMp1409841)

16 Ahn HS \& Welch HG. South Korea's thyroid-cancer "epidemic": turning the tide. New England Journal of Medicine 2015373 2389-2390. (doi:10.1056/NEJMc1507622) 
17 Ito Y, Miyauchi A, Kihara M, Higashiyama T, Kobayashi K \& Miya A. Patient age is significantly related to the progression of papillary microcarcinoma of the thyroid under observation. Thyroid 201424 27-34. (doi:10.1089/thy.2013.0367)

18 Lang BH \& Wong CK. A cost-effectiveness comparison between early surgery and non-surgical approach for incidental papillary thyroid microcarcinoma. European Journal of Endocrinology 2015173 367-375. (doi:10.1530/EJE-15-0454)

19 Kwak JY. Indications for fine needle aspiration in thyroid nodules. Endocrinology and Metabolism 201328 81-85. (doi:10.3803/ EnM.2013.28.2.81)

20 Haugen BR, Alexander EK, Bible KC, Doherty GM, Mandel SJ, Nikiforov YE, Pacini F, Randolph GW, Sawka AM, Schlumberger M et al. 2015 American thyroid association management guidelines for adult patients with thyroid nodules and differentiated thyroid cancer. Thyroid 201626 1-133. (doi:10.1089/thy.2015.0020)

21 Han JM, Kim WG, Kim TY, Jeon MJ, Ryu JS, Song DE, Hong SJ, Shong YK \& Kim WB. Effects of low-dose and high-dose postoperative radioiodine therapy on the clinical outcome in patients with small differentiated thyroid cancer having microscopic extrathyroidal extension. Thyroid 201424 820-825. (doi:10.1089/thy.2013.0362)

22 Jeon MJ, Kim WG, Choi YM, Kwon H, Song DE, Lee YM, Sung TY, Yoon JH, Hong SJ, Baek JH et al. Recent changes in the clinical outcome of papillary thyroid carcinoma with cervical lymph node metastasis. Journal of Clinical Endocrinology and Metabolism 2015100 3470-3477. (doi:10.1210/JC.2015-2084)

23 Lang BH, Chan DT, Chow FC, Wong KP \& Chang RY. The association of discolored parathyroid glands and hypoparathyroidism following total thyroidectomy. World Journal of Surgery 201640 1611-1617. (doi:10.1007/s00268-016-3462-9)

24 Bollerslev J, Rejnmark L, Marcocci C, Shoback DM, Sitges-Serra A, van Biesen W, Dekkers OM \& European Society of Endocrinology. European society of endocrinology clinical guideline: treatment of chronic hypoparathyroidism in adults. European Journal of Endocrinology 2015173 G1-G20. (doi:10.1530/EJE-15-0628)
25 Bilimoria KY, Bentrem DJ, Ko CY, Stewart AK, Winchester DP, Talamonti MS \& Sturgeon C. Extent of surgery affects survival for papillary thyroid cancer. Annals of Surgery 2007246 375-381; discussion 381-374. (doi:10.1097/SLA.0b013e31814697d9)

26 Mazzaferri EL \& Kloos RT. Clinical review 128: current approaches to primary therapy for papillary and follicular thyroid cancer. Journal of Clinical Endocrinology and Metabolism 200186 1447-1463. (doi:10.1210/jcem.86.4.7407)

27 American Thyroid Association Guidelines Taskforce on Thyroid Nodules and DifferentiatedThyroid Cancer, Cooper DS, Doherty GM, Haugen BR, Kloos RT, Lee SL, Mandel SJ, Mazzaferri EL, McIver B, Pacini F et al. Revised american thyroid association management guidelines for patients with thyroid nodules and differentiated thyroid cancer. Thyroid 200919 1167-1214. (doi:10.1089/thy.2009.0110)

28 Nixon IJ, Ganly I, Patel SG, Palmer FL, Whitcher MM, Tuttle RM, Shaha A \& Shah JP. Thyroid lobectomy for treatment of well differentiated intrathyroid malignancy. Surgery 2012151 571-579. (doi:10.1016/j.surg.2011.08.016)

29 Barney BM, Hitchcock YJ, Sharma P, Shrieve DC \& Tward JD. Overall and cause-specific survival for patients undergoing lobectomy, neartotal, or total thyroidectomy for differentiated thyroid cancer. Head and Neck 201133 645-649. (doi:10.1002/hed.21504)

30 Haigh PI, Urbach DR \& Rotstein LE. Extent of thyroidectomy is not a major determinant of survival in low- or high-risk papillary thyroid cancer. Annals of Surgical Oncology 200512 81-89. (doi:10.1007/ s10434-004-1165-1)

31 Kandil E, Krishnan B, Noureldine SI, Yao L \& Tufano RP. Hemithyroidectomy: a meta-analysis of postoperative need for hormone replacement and complications. Journal of Oto-Rhino-Laryngology and Its Related Specialties 201375 6-17. (doi:10.1159/000345498)

32 Hauch A, Al-Qurayshi Z, Randolph G \& Kandil E. Total thyroidectomy is associated with increased risk of complications for low- and highvolume surgeons. Annals of Surgical Oncology 201421 3844-3852. (doi:10.1245/s10434-014-3846-8)

Received 8 October 2016

Revised version received 3 January 2017

Accepted 12 January 2017 